\title{
Platelets in Thrombo-Inflammation: Concepts, Mechanisms, and Therapeutic Strategies for Ischemic Stroke
}

\author{
Philipp Burkard ${ }^{1}$ Timo Vögtle ${ }^{1}$ Bernhard Nieswandt ${ }^{10}$ \\ ${ }^{1}$ Institute of Experimental Biomedicine, University Hospital and \\ Rudolf Virchow Center, University of Würzburg, Würzburg, Germany \\ Hämostaseologie 2020;40:153-164.

\begin{abstract}
Address for correspondence Bernhard Nieswandt, PhD, Institute of Experimental Biomedicine I, University Hospital Würzburg and Rudolf Virchow Center for Experimental Biomedicine, University of Würzburg, Josef-Schneider-Str. 2, 97080 Würzburg, Germany
\end{abstract} \\ (e-mail: bernhard.nieswandt@virchow.uni-wuerzburg.de).
}

\author{
Abstract \\ Keywords \\ - platelet \\ - platelet receptors \\ - stroke \\ - thrombo- \\ inflammation \\ - FXII
}

Zusammenfassung
Platelets are anucleate cells known for their essential function in hemostasis and formation of thrombi under pathologic conditions. In recent years, strong evidence emerged demonstrating the critical involvement of platelets in inflammatory processes including acute ischemic stroke (AIS), which is one of the leading causes of death and disability worldwide. Recanalization of the occluded brain artery to reconstitute cerebral blood flow is the primary goal in the treatment of stroke patients. However, despite successful reperfusion many patients show progression of infarct sizes, a phenomenon referred to as ischemia/reperfusion injury (I/RI). Cerebral I/RI involves both thrombotic as well as inflammatory pathways acting in concert to cause tissue damage, defining AIS as a prototypic thrombo-inflammatory disease. Currently used antiplatelet drugs applied to AIS patients eventually increase the risk of partially lifethreatening hemorrhages, making more targeted pharmacological intervention necessary. Experimental evidence indicates that inhibition of platelet surface receptors that regulate initial platelet adhesion and activation might be suitable targets in thrombo-inflammatory settings, while inhibitors of platelet aggregation are not. In this review, we will summarize the recent developments in elucidating the role of the main platelet receptors in AIS and discuss their potential as pharmaceutical targets. Furthermore, we will also briefly discuss the important platelet-triggered intrinsic coagulation pathway with the pro-inflammatory kallikrein-kinin system in the context of ischemic stroke.

Thrombozyten sind anukleäre Zellen, die für ihre entscheidende Bedeutung in der Hämostase, aber auch für die Bildung von gefäßverschließenden Thromben unter pathologischen Bedingungen bekannt sind. In den letzten Jahren belegten jedoch einige Studien auch eine wichtige Rolle von Thrombozyten bei inflammatorischen Prozessen. Zu diesen gehört auch der ischämische Schlaganfall (acute ischemic stroke AIS) - eine der häufigsten Todesursachen und Verursacher von Behinderungen weltweit. Bei dessen Behandlung ist das primäre Ziel die Rekanalisierung des verschlossenen Gefäßes, durch die der Blutfluss wiederhergestellt wird. Dennoch vergrößern sich bei vielen Patienten trotz erfolgreicher Reperfusion des betroffenen Areals die received

December 17, 2019

accepted after revision

March 18, 2020
(C) 2020 Georg Thieme Verlag KG Stuttgart . New York
DOI https://doi.org/

10.1055/a-1151-9519.

ISSN 0720-9355. 
Schlüsselwörter

- Thrombozyten

- Thrombozytenrezeptoren

- Schlaganfall

- Thromboinflammation

- FXII
Infarktgebiete weiter. Dieses Phänomen wird Ischämie-Reperfusionsschaden (ischemia / reperfusion injury - I/RI) genannt. Bei dem zerebralen I/RI sind ineinandergreifende prothrombotische als auch inflammatorische Mechanismen an der Entstehung von Gewebeschäden beteiligt - AIS gilt daher als Prototyp thrombo-inflammatorischer Erkrankungen. Der Einsatz von gängigen Thrombozytenfunktionshemmern in AIS Patienten führt häufig zu - in Einzelfällen lebensgefährlichen - Blutungen, was die Notwendigkeit einer neuen, zielgerichteteren pharmakologischen Intervention verdeutlicht. Experimentelle Daten zeigen, dass die Inhibition von Thrombozytenrezeptoren, die an der initialen Adhäsion und Aktivierung der Zellen beteiligt sind, möglicherweise gute Angriffspunkte zur Behandlung thrombo-inflammatorischer Erkrankungen darstellen - klassische Thrombozytenaggregationshemmer jedoch nicht. In diesem Übersichtsartikel fassen wir die neuesten Entwicklungen der Forschung über die Funktion der wichtigsten Thrombozytenrezeptoren im AIS zusammen und diskutieren deren Eignung als neue pharmakologische Zielstrukturen. Darüber hinaus diskutieren wir kurz die Rolle der von Thrombozyten induzierten Kontaktaktivierung im AIS, die Startpunkt der intrinsischen Kaogulationskaskade und des inflammatorischen Kallikrein-Kinin-Systems ist.

\section{Introduction}

Platelets are essential players in hemostasis and contribute to the maintenance of vascular integrity under physiological conditions. Dysregulated platelet activation and aggregation in combination with local coagulation can cause formation of vessel-occluding thrombi under pathological conditions, resulting in ischemia and infarction of affected tissues. Depending on the site of thrombus formation, this can cause life-threatening disease states such as myocardial infarction, deep vein thrombosis, potentially leading to pulmonary embolism or stroke. ${ }^{1-4}$ Besides the well-known functions in hemostasis and thrombosis, platelets got into focus in the context of inflammatory disease processes, like host defense or post-ischemic tissue damage in multiple organ systems. For example, platelets are known to be involved in leukocyte recruitment to sites of inflammation by direct interaction with inflammatory cells as well as the release of pro-inflammatory cytokines. ${ }^{5,6}$ Moreover, other substances released by platelets are able to mediate the generation of pro-inflammatory bradykinin via the kallikrein-kinin pathway. These atypical platelet functions, linking thrombotic cascades to innate immunity, are based on essentially different mechanisms than those utilized in normal hemostasis and are involved in pathological processes now referred to as thrombo-inflammation, a word that was initially coined to describe the pathomechanisms that drive neuronal damage in the setting of ischemic stroke. ${ }^{7}$

Amongst all neurological diseases, stroke accounts for the largest proportion of disability and deaths worldwide. Only $20 \%$ of all strokes are caused by intracranial hemorrhage (ICH). The majority is due to cerebral ischemia, caused by a thromboembolic occlusion of a major cerebral artery, leading to acute ischemic stroke (AIS). ${ }^{8,9}$ Thus, the primary goal of therapy is the recanalization of occluded vessels to restore cerebral blood flow. This is achieved either by chemical thrombolysis with the tissue plasminogen activator (tPA) or, more recently, by mechanical thrombectomy (MT). Although fast intervention by either of the techniques increased the rate of restored blood flow markedly, the number needed to treat to benefit (NNTB) is still high. Within the first 3 to 4.5 hours after ischemic stroke, the NNTB for thrombolytic treatment with TPA is around 7 and around 2 for MT, which is most likely a consequence of the higher recanalization rates achieved by $\mathrm{MT}$, demonstrating the necessity of restoring blood flow. ${ }^{10}$ This is also in line with the observation that patients neither eligible for pharmacological nor mechanical removal of the thrombus or in which the therapeutic procedure fails, occlusion persists, and infarct volume rapidly increases. ${ }^{11,12}$ This pathological state is known as permanent ischemia. The eligibility of pharmacological recanalization is dependent on different factors and exclusion criteria, e.g., the stage of infarct development, previous ICH, low platelet count $(<100,000 / \mu \mathrm{L})$, or myocardial infarction in the last 3 months. ${ }^{11}$ Although MT is an effective alternative for patients ineligible for tPA treatment, absence of proximal intracranial occlusion, hospital arrival outside the eligible time window, low baseline National Institutes of Health Stroke Scale (NIHSS)score, and posterior circulation cerebral ischemia are the four most common contraindications for MT. ${ }^{13}$ However, even despite successful recanalization, infarct sizes often continue to grow, and the underlying molecular mechanisms are only poorly understood. This phenomenon is referred to as ischemia/reperfusion injury (I/RI) and can also be seen in other organ systems like heart, liver, and kidney. ${ }^{14-16}$ Such infarct growth after successful recanalization is also prominently observed in experimental models of stroke, most notably in the transient middle cerebral artery occlusion (tMCAO) model. This model utilizes a very thin filament, which is brought into the middle cerebral artery (MCA) via the internal carotid artery to induce vessel occlusion for a defined time period (typically 
60 minutes) followed by its removal, thereby mimicking the pathology of ischemia due to thromboembolism with subsequent reperfusion injury after recanalization. ${ }^{17}$

Permanent ischemia and I/RI in hospitalized stroke patients have been tightly connected to neuro-inflammatory processes including the infiltration of immune cells, especially $\mathrm{T}$ cells, into the infarcted brain tissue. This has been observed in experimental models of stroke as well as in human patients. ${ }^{7,18-21}$ In addition, neutrophilic granulocytes, the first immune cells appearing at sites of inflammatory brain injuries, are implicated in further damaging the blood-brain barrier by releasing proteolytic enzymes, reactive oxygen species, and neutrophil extracellular traps (NETs). Notably, the interaction of platelets with neutrophilic granulocytes has been shown to induce the formation of NETs in various inflammatory conditions, and might also play a role in AIS. ${ }^{22,23}$ The exact contribution of neutrophilic granulocytes to ischemic brain damage and I/RI, however, is not fully elucidated and are controversially discussed (reviewed in Strecker et al. ${ }^{24}$ ).

Already in the 1980s, the accumulation of platelets and factors of the coagulation system at sites of cerebral infarction was recognized. ${ }^{25,26}$ The contribution of platelets and infiltrating immune cells, especially $\mathrm{T}$ cells, to the progression of cerebral I/RI is now supported by several experiments. ${ }^{19}$ The finding that the closely intertwined action of thrombotic and inflammatory mechanisms drives post-ischemic cerebral infarct progression leads to the concept of ischemic stroke being a thrombo-inflammatory disease. Strikingly, the treatment of patients with classical antiplatelet drugs like aspirin or clopidogrel after recanalization does not reliably reduce infarct growth in all patients, probably because they have only limited effects on thrombo-inflammatory processes. In contrast, these treatments emerged as rather unfavorable, since they often result in $\mathrm{ICH} .{ }^{27,28}$ Hence, more targeted pharmacological intervention to limit thrombo-inflammatory activity of platelets is needed to prevent patients from I/RI while minimizing the risk of ICH.

In this review we will summarize physiological functions of the main platelet receptors and then discuss their role in the thrombo-inflammatory disease setting of AIS and their potential as pharmaceutical targets, based on experimental and, in part, clinical studies. Furthermore, we will briefly discuss the connection between the important platelettriggered factor XII (FXII)-driven intrinsic coagulation pathway and the pro-inflammatory kallikrein-kinin system (KKS) in the context of ischemic stroke, emphasizing the direct involvement of platelets in mediating inflammatory processes by inducing the production of the pro-inflammatory compound bradykinin.

\section{Platelets and Hemostasis}

At sites of vascular injury, the first contact of platelets with the exposed extracellular matrix (ECM) is mediated by the receptor complex glycoprotein (GP)Ib-V-IX ( - Fig. 1). This receptor complex, more precisely its GPIb $\alpha$ subunit, interacts with von Willebrand factor ( $v W F$ ) which derives from endothelial cells or is released from the $\alpha$-granules of platelets and is then captured at sites of endothelial damage or activation. ${ }^{29,30}$ This first interaction leads to an initial tethering and deceleration of

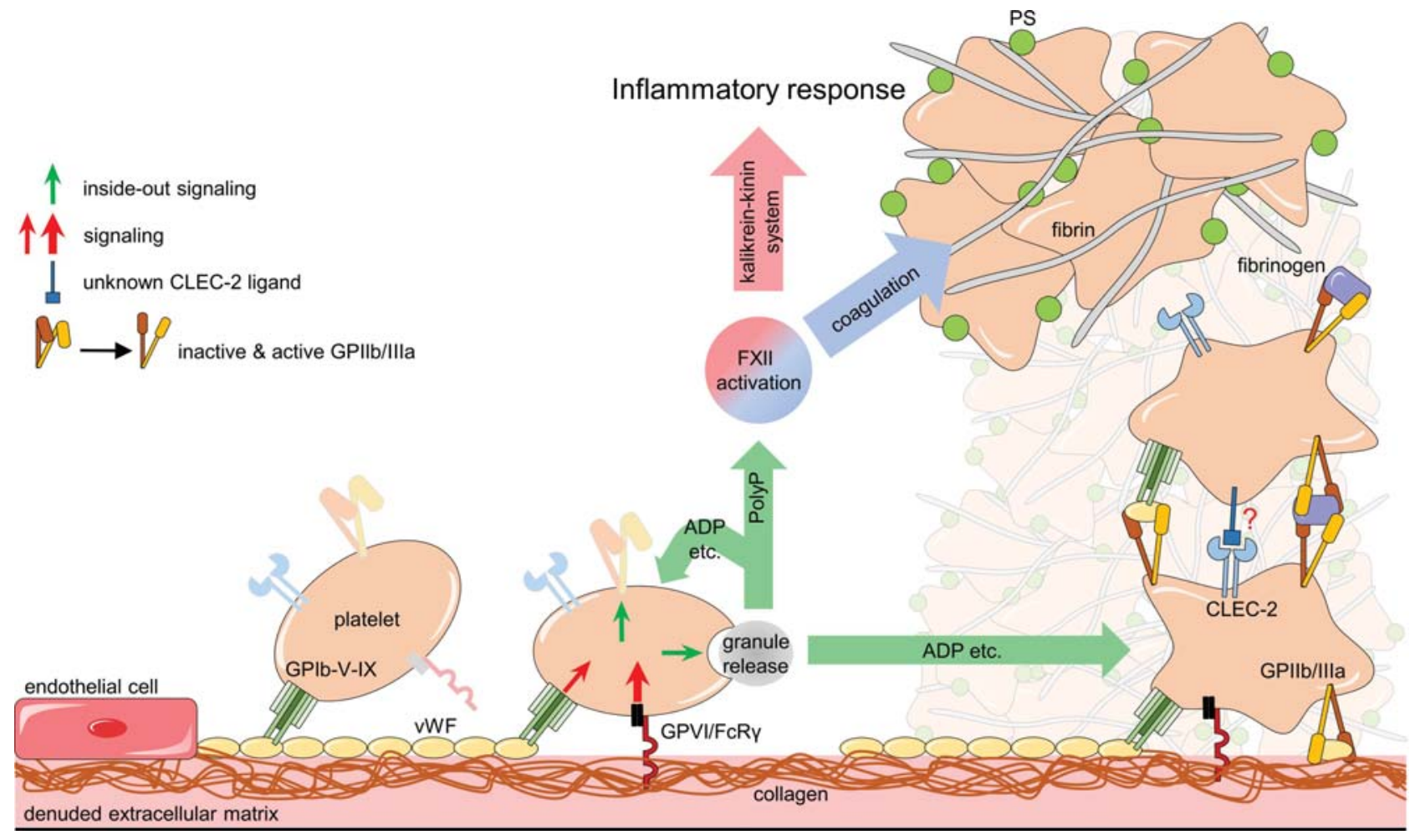

Fig. 1 Model of platelet adhesion and activation at the site of vascular injury and subsequent thrombus formation. The scheme highlights the main platelet receptors involved in platelet tethering (GPIb), activation (GPVI), firm adhesion and thrombus growth (CLEC-2, GPIIb/IIla), and the connection to the intrinsic coagulation system (FXII) with the pro-inflammatory kallikrein-kinin system (for details see the text). 
the platelets under high shear as prevailing, e.g., in stenosed arteries, but does by itself not mediate firm adhesion or thrombus growth. ${ }^{31}$ However, this slower "rolling" along the vessel wall allows other receptors that mediate platelet activation to interact with their corresponding ligands. One of those is the immunoglobulin-like receptor GPVI, which binds to collagen, but also other ligands such as fibrin. ${ }^{32-34}$ Due to its low affinity for collagen, this interaction does not lead to stable adhesion by itself but rather mediates cellular activation via the immunoreceptor tyrosine-based activation motif (ITAM) in the associated $\mathrm{Fc}$ receptor $(\mathrm{FCR}) \gamma$-chain, triggering a tyrosine phosphorylation cascade which leads to activation of effector enzymes and $\mathrm{Ca}^{2+}$ mobilization. ${ }^{33,35,36}$ These processes result in the activation of the platelets and the release of secondary mediators like adenosine diphosphate (ADP) and serotonin as well as the production of thromboxane $\mathrm{A}_{2}\left(\mathrm{TxA}_{2}\right.$; - Fig. 1). Additionally, platelet activation results in scramblase-mediated exposure of negatively charged phosphatidylserine on the platelet surface, providing a platform for the assembly of two major coagulation factor complexes and subsequent thrombin production. ${ }^{37}$ Simultaneously exposed tissue factor contributes to initiates the local generation of thrombin. Together with ADP and $\mathrm{TxA}_{2}$, thrombin further augments platelet activation via signaling through G-protein coupled receptors. ${ }^{38}$ Another major activation receptor is the hem-ITAM bearing C-type lectin-like receptor 2 (CLEC-2), of which the only known endogenous ligand is podoplanin, a membrane glycoprotein not normally expressed within the vasculature. ${ }^{33,39}$ All these outside-in signals result in the conformational change of the most prominent adhesion receptor in platelets, integrin $\alpha \mathrm{IIb} \beta 3$ (GPIIb/IIIa) from an inactive to a high affinity state for its multiple ligands, leading to firm platelet adhesion, aggregation, and thrombus formation. Another consequence of platelet activation is the release of inorganic polyphosphate (PolyP) triggering activation of coagulation factor XII (FXII) which represents the starting point of the intrinsic coagulation pathway and the KKS ( - Fig. 1). ${ }^{40}$

\section{Targeting GPIIb/IIla in Ischemic Stroke}

Once platelets are activated through their surface agonist receptors, an inside-out signaling cascade leads to a conformational change of GPIIb/IIIa from a low affinity to an active high affinity binding state, which allows firm adhesion to the ECM by binding multiple ligands such as vWF, fibronectin, and vitronectin. Furthermore, GPIIb/IIIa facilitates aggregate formation by bridging neighboring platelets through fibrinogen and vWF ( - Fig. 1). ${ }^{2}$ Its essential role in thrombus formation suggests that GPIIb/IIIa might be an attractive target to combat acute ischemic disorders. Currently, there are three pharmacological inhibitors for GPIIb/IIIa used in the clinical routine: the chimeric humanized monoclonal antibody fragment 7E3 Fab abciximab, eptifibatide, a KGD-based heptapeptide, and tirofiban, which is a nonpeptide fibrinogen mimetic. ${ }^{41}$ The beneficial effect of GPIIb/IIIa inhibition is well established in acute coronary syndrome patients undergoing percutaneous coronary intervention, where I/RI is a known pathological process leading to increased infarct sizes as well. ${ }^{42,43}$ In a study where rat hearts were subjected to I/RI and transfused with platelets from patients with acute myocardial infarction (AMI) ex vivo, it was shown that those hearts which received platelets from AMI patients co-incubated with abciximab developed less myocardial injury after ischemia and reperfusion than those receiving untreated platelets. ${ }^{44}$ Also in patients with AMI, the clinical outcome is markedly improved when abciximab is applied in combination with heparin or mechanical removal of the thrombus compared with patients who are not co-treated with abciximab. ${ }^{45,46}$ These findings suggest that $\mathrm{I} / \mathrm{RI}$ in AMI is worsened by activated platelets and anti-GPIIb/IIIa treatment attenuates these effects. In the case of ischemic stroke, however, the therapeutic potency of GPIIb/IIIa-targeting drugs is discussed controversially. Early experimental studies utilizing the TMCAO model in mice and a thromboembolic model in rabbits, where a blood clot was injected into the cerebral arterial system, showed a beneficial effect of GPIIb/IIIa blockade by the nonpeptide SDZ GPI 562 and the synthetic cyclic nonpeptides TP9201 and SM-20302 on neurological outcome and infarct sizes in the setting of ischemic stroke. Strikingly, these studies showed that GPIIb/IIla inhibition even led to a dampened hemorrhagic effect of tPA treatment or exhibited no ICH in therapeutic doses. ${ }^{47-49}$ As a potential explanation for this surprising finding, the authors suggested that this might be due to the prevention of reoccurring microthrombi when GPIIb/IIIa was blocked. Contradictory to that, mouse models of tMCAO revealed that inhibition of GPIIb/IIIa by $\mathrm{F}(\mathrm{ab})_{2}$ fragments of the function-blocking antibody JON/A leads to severe ICH and eventually to death of the treated mice. More importantly, however, those mice that did not develop severe ICH showed similar infarct sizes to control-treated animals regardless of the dose of antibody. Furthermore, these effects held true when older mice or mice with certain comorbidities were subjected to the same experimental setup. ${ }^{50,51}$ Similar consequences of antiGPIIb/IIIa administration were observed in different clinical studies as well. In an international, randomized, placebocontrolled, double-blind study, the administration of abciximab led to severe intracranial bleeding in a significant number of patients after 5 days regardless of the timepoint of intravenous injection after stroke, onset of stroke, or wake-up stroke. Thus, the study was terminated in phase III after inclusion of 808 patients ( - Table 1 ). ${ }^{52}$

Tirofiban is frequently used to prevent vessel re-occlusion after mechanical removal of thrombi or stenting, which can cause vascular damage. However, a prospective study of patients with ischemic stroke revealed that those receiving tirofiban had significantly higher risks of intracranial bleeding and poor outcome compared with control. ${ }^{53}$ Nonetheless, especially tirofiban is discussed controversially regarding its use in AIS patients, since some studies showed the safety and efficacy of this drug in patients with rescue stenting, using tirofiban alone or together with standard thrombolytic therapy. ${ }^{54,55}$ In conclusion, despite the high potency of anti-GPIIb/IIIa treatment in patients with AMI and some promising clinical studies using tirofiban in a certain subgroup of ischemic stroke patients, this receptor has to be 


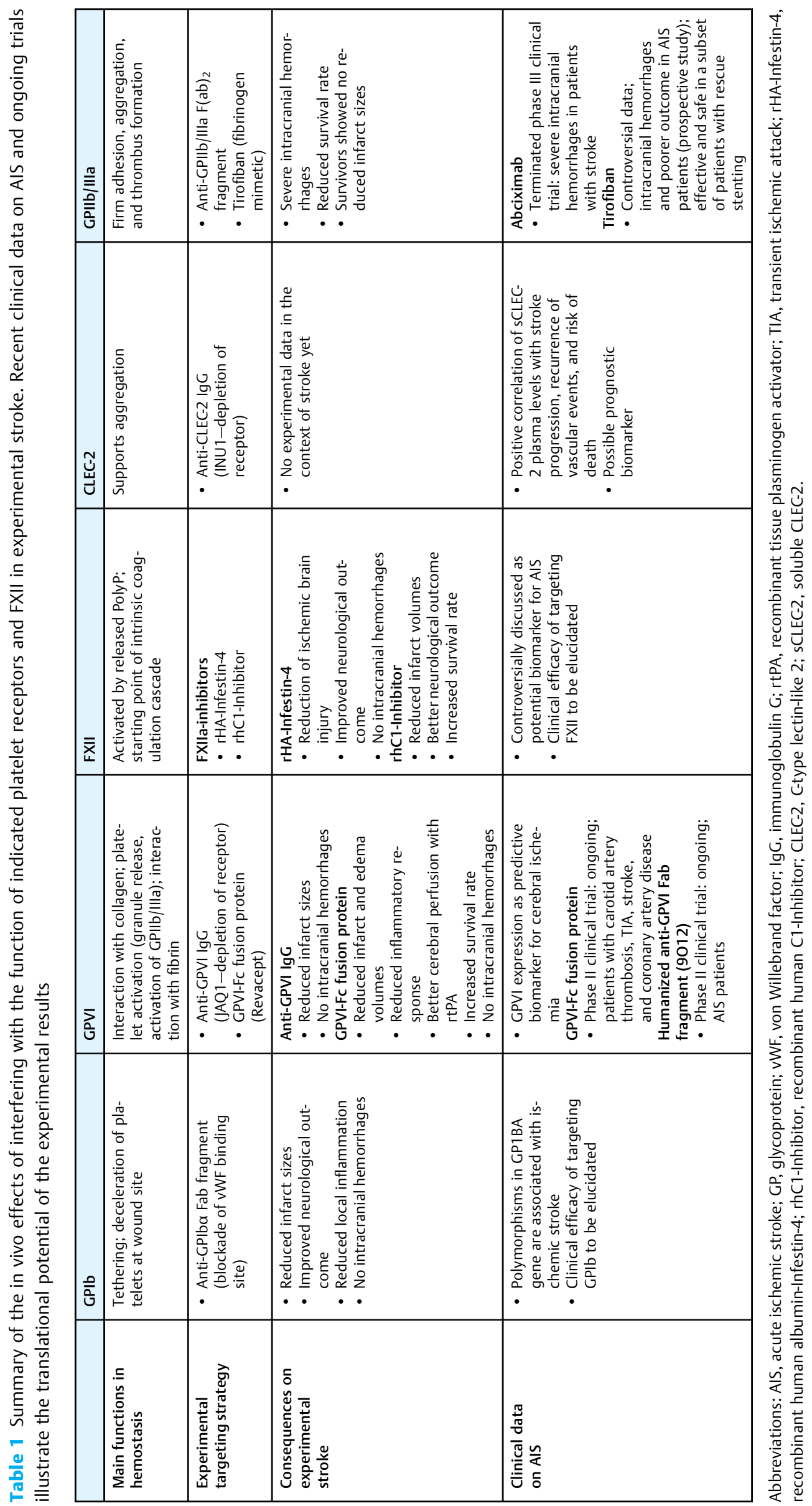


considered as unreliable, yet druggable target in the therapy of ischemic stroke (-Table 1). Very importantly, these data provide strong evidence that platelet aggregation may not be of major significance for thrombo-inflammation and immune cell recruitment in the infarcted brain, implicating that other platelet-dependent mechanisms are responsible for the pathological progress of $\mathrm{I} / \mathrm{RI}$ in AIS.

\section{Targeting GPIba-vWF Interaction in Ischemic Stroke}

The subunit GPIb $\alpha$ of the GPIb-V-IX receptor complex directly binds to immobilized vWF but also multiple other ligands such as P-selectin and macrophage antigen 1 (Mac-1), which can be found on the surface of immune cells, e.g., neutrophilic granulocytes. Furthermore, components of the coagulation cascade like FXI, FXII, and FIIa (thrombin) are known ligands of GPIba. ${ }^{2}$ In experimental models of tMCAO, the blockade of GPIb $\alpha$ by intravenous injection of Fab-fragments of the function-blocking antibody $\mathrm{p} 0 \mathrm{p} / \mathrm{B}$, reduced infarct sizes to approximately $40 \%$ compared with control animals. This protective effect was also reflected by an improved neurological outcome and sustained reperfusion, while no intracerebral hemorrhages were observed ( - Table 1). ${ }^{50,56}$ Notably, these beneficial effects were observed regardless of whether the treatment was given 1 hour before or 1 hour after tMCAO, indicating not only a protective but also a potential therapeutic effect of GPIb $\alpha$ blockade.$^{50}$ In addition, another study utilizing the tMCAO model demonstrated that p0p/B Fab treatment attenuates the local inflammation as shown by reduced monocyte and CD4 T cell infiltration into the infarcted brain and diminished expression of pro-inflammatory cytokines like interleukin (IL) 6 , IL- $1 \beta$, and tumor necrosis factor- $\alpha .{ }^{57}$ Similar results were obtained using a mouse model, where the ectodomain of GPIb $\alpha$ is replaced by the ectodomain of the human IL-4 receptor (IL4R $\alpha / G P I b \alpha)$ leading to abolished ligand binding without the loss of the receptor complex..$^{58,59}$ These findings emphasize the relevance of GPIb $\alpha$ for the progression of I/RI in stroke. Conversely, also mice lacking the main ligand of GPIb $\alpha$, vWF, developed smaller infarcts and displayed a better neurological outcome in the tMCAO model as compared with their respective controls without showing signs of ICH. Of note, reconstitution of plasma vWF by hydrodynamic gene transfer was sufficient to revert the protection, showing that these results were specifically attributable to the loss of plasma vWF. ${ }^{60}$ Furthermore, vWF derived from platelets in chimeric mice has been shown to be sufficient to revert the protective effect of vWF deficiency in the tMCAO model, showing that even a small fraction of vWF is sufficient to increase infarct volumes and worsen neurological outcome. ${ }^{61}$ Other studies focused on the protease A disintegrin and metalloprotease with thrombospondin type 1 repeats 13 (ADAMTS13), which cleaves $\mathrm{VWF}$ into smaller, less active fragments thereby modulating the total activity of vWF. Mice deficient in ADAMTS13 (Adamts $13^{-I-}$ ) showed significantly increased infarct volumes and worsened neurological outcome after tMCAO ${ }^{62,63}$ In line with these results, treatment of wild-type mice with recombinant human ADAMTS13 (r-hu-ADAMTS13) before reperfu- sion resulted in reduced infarct volumes compared with untreated animals after experimental cerebral ischemia, indicating that ultra-large vWF multimers are of particular pathogenic relevance for thrombo-inflammatory damage in the acutely ischemic brain. ${ }^{62}$

The intracellular signaling cascade triggered by GPIb $\alpha-v W F$ interactions is not fully understood. It is known that the intracellular domain of GPIb is associated with the actinbinding protein $14-3-3 \zeta$ and various signaling molecules like phosphatidylinositol kinase 3 (PI3K), Src-related tyrosine kinases, GTPase-activating proteins, and tyrosine phosphatases have been proposed to be activated by ligand binding to GPIb $\alpha$. This downstream signaling is reported to induce weak intracellular $\mathrm{Ca}^{2+}$ mobilization and activation of $\alpha$ IIbß3 integrin. ${ }^{64}$ The lipid signaling enzyme phospholipase D 1 (PLD1) may play a key role in GPIb-dependent platelet activation since platelets derived from mice deficient for PLD1 $\left(\right.$ Pld $\left.^{-1^{-}}{ }^{-}\right)$ showed defective adhesion on vWF-coated surfaces under high shear conditions. ${ }^{65}$ Furthermore, it has been shown that PLD1 deficiency resulted in reduced platelet-dependent leukocyte recruitment under inflammatory conditions in in vivo models of carotid artery injury and venous thrombosis. ${ }^{66}$ The significant role of both PLD isoforms, PLD1 and PLD2, for thrombus formation was reflected by defective $\alpha$-granule release and prevention of vessel occlusion in a model of $\mathrm{FeCl}_{3}$-induced arteriolar thrombosis in mice deficient for both isoforms. ${ }^{67}$ Moreover, pharmacological inhibition of PLD with 5-fluoro-2-indolyldes-chlorohalopemide (FIPI) in mice leads to delayed thrombus formation and vessel occlusion in two in vivo models of arterial thrombosis, the $\mathrm{FeCl}_{3}$-induced injury of the carotid artery, and the mesenteric artery thrombosis model, suggesting an antithrombotic effect of FIPI. Importantly, FIPI-treated mice showed significantly reduced infarct volumes, ischemic lesions, and better neurological outcome after subjection to tMCAO compared with untreated mice, without developing intracerebral hemorrhages, pointing to a promising and safe treatment of AIS. ${ }^{68}$ Besides evidence of the role of GPIb-vWF interactions and resulting signaling in murine models of ischemic stroke, studies in human stroke patients support the experimental data ( - Table 1$)$. There are several known genetic variations in the GP1BA gene. The $-5 \mathrm{~T} / \mathrm{C}$ dimorphism in the untranslated Kozak sequence has been recognized for altering the expression level of GPIb $\alpha$. In addition, a Thr/Met change in the coding region and a variable number of tandem repeat sequence have been shown to affect the protein structure. All of them have been associated with the development of ischemic stroke in patients carrying certain variants of the mentioned polymorphisms. ${ }^{69}$

\section{Targeting GPVI in Ischemic Stroke}

GPVI is the main MK/platelet collagen receptor and mediates cellular activation of platelets. Ligand binding initiates signaling through the receptor-associated FcR $\gamma$-chain dimers, which bear an ITAM. This results in rapid rearrangement of the cytoskeleton, $\mathrm{Ca}^{2+}$ mobilization, granule release, and activation of integrin $\alpha$ IIbß3 (GPIIbIIIa). ${ }^{33,70}$ In mice, the in vivo application of the monoclonal anti-GPVI antibody JAQ1 leads 
to the depletion, i.e., irreversible loss of GPVI in circulating platelets. This results in a GPVI knockout-like phenotype and abolished collagen responses for at least two weeks. ${ }^{71}$ Using intravital fluorescence microscopy in a model of carotid artery injury in mice, Massberg et al. showed that GPVI depletion (JAQ1 IgG) or its functional inhibition (JAQ1 Fab) resulted in reduced platelet tethering at the injured vessel wall and significantly diminished stable platelet capture and aggregation. ${ }^{72}$ Furthermore, platelets of GPVI-depleted mice failed to adhere to collagen under static as well as under flow conditions and the animals were protected from experimental lethal pulmonary thromboembolism. ${ }^{73}$ More recently, two studies have demonstrated that GPVI also interacts with fibrin, thereby contributing to thrombus growth and stabilization. ${ }^{32,34}$ Altogether, these studies highlight GPVI as one major receptor in arterial thrombus formation. In the tMCAO model, GPVI depletion leads to a significant reduction of infarct volumes, although to a lesser extent than GPIba inhibition (p0p/B-Fab). ${ }^{50}$ Strikingly, no ICH was observed in $G P 6^{-I-}$ or GPVI-depleted mice, indicating that GPVI might be a promising pharmacological target for the treatment of acute stroke. This is supported by a very recent study utilizing the tMCAO model, in which mice were treated with JAQ1 and concomitantly received human recombinant $\mathrm{PA}(\mathrm{rtPA})$, the standard treatment of ischemic stroke patients. Of note, depletion of GPVI in this co-treatment still exhibited its protective effect on infarct progression and improved neurological outcome in the acute phase after tMCAO, but caused no bleeding complications $(-$ Table 1$){ }^{74}$

Ligand binding triggers cross-linking of GPVI, bringing its FcRr chains and the Src family tyrosine kinases (SFKs) Fyn and Lyn into proximity. This initiates a tyrosine phosphorylation cascade starting with the spleen tyrosine kinase (Syk), which in turn phosphorylates the adapter protein linker for activation of T cells (LAT) and finally leads to the activation of effector enzymes, most notably PL $\mathrm{C} \gamma 2$ and phosphoinositide 3-kinase (PI3K). ${ }^{33,38}$ In the tMCAO model, mice genetically lacking Syk in platelets (Syk ${ }^{\mathrm{fl} / \mathrm{fl}, \mathrm{Pf} 4-\mathrm{cre}+/-}$ ) showed significantly reduced infarct sizes and better neurological scores compared with the corresponding control. Notably, the same outcome was observed in wild-type mice after oral administration of the Syk inhibitor, Bl1002494, which exhibited its protective effect also when administered after removal of the filament, i.e., during the reperfusion phase. In both cases, no intracerebral hemorrhages were observed. Hence, pharmacological Syk inhibition appears to be a potentially safe therapeutic intervention to protect patients from arterial thrombosis and infarct growth after successful recanalization and restored perfusion. ${ }^{75}$

Src-like adapter proteins (SLAP and SLAP2) are structurally similar to SFKs but lack the kinase domain and seem to inhibit ITAM-receptor-mediated platelet activation, since mice deficient for both proteins exhibited platelet hyperresponsiveness upon GPVI stimulation in vitro. ${ }^{76}$ In line with the neurological protective effects of GPVI blockade or inhibition of downstream signaling molecules, genetically SLAP1/2 double-deficient mice displayed markedly increased infarct volumes and worsened neurological outcomes after tMCAO. ${ }^{76}$
The absence of notable bleeding phenotypes when interfering with GPVI-ITAM signaling in the TMCAO model of ischemic stroke suggests that GPVI might be a potent and safe therapeutic target to prevent cerebral infarct progression in patients with successful recanalization ( - Table 1 ). A possible role of GPVI in acute stroke in humans was shown in a cohort study including patients with transient ischemic attack (TIA) and stroke as well as patients with non-ischemic events as controls. ${ }^{77}$ Here, the authors showed that GPVI expression of platelets from TIA and stroke patients was significantly elevated compared with controls. Furthermore, they revealed a positive correlation between GPVI levels and relative risk of stroke and, notably, a poorer clinical outcome. By providing a cut-off value of GPVI expression, they suggested GPVI as a predictive biomarker for the occurrence of cerebral ischemia.

GPVI expression can be downregulated by ectodomain shedding by the metalloproteinases ADAM10 and ADAM17, thereby releasing soluble GPVI (sGPVI) into the blood plasma. ${ }^{78,79}$ A clinical study showed significantly increased plasma levels of sGPVI in patients with acute stroke, implicating GPVI shedding in the acute phase of the disease.$^{80}$ Revacept, a dimeric GPVI-Fc fusion protein, which inhibits the GPVIcollagen interaction, is the first clinically tested GPVI inhibitor. ${ }^{81}$ Under conditions of ischemic stroke, Revacept treatment in mice resulted in better cerebral reperfusion when given with concomitant rtPA injection. Furthermore, the compound improved neurological score and survival rate and also decreased infarct and edema volumes compared with control animals without causing intracranial bleeding. ${ }^{82}$ Additionally, several indicators of inflammatory cell infiltration and reperfusion damage were reduced upon Revacept treatment, e.g., the detection of immunoglobulin $\mathrm{G}(\mathrm{IgG})$, infiltrated macrophages, transforming growth factor $\beta$, and platelet-derived growth factor in brain sections. ${ }^{82}$ Currently, Revacept is being tested in phase II clinical trials in patients with carotid artery stenosis, TIA, or stroke (NCT 01645306) and in patients with coronary artery disease (NCT 03312855; - Table 1). Another promising pharmacological compound interfering with GPVI function is the humanized Fab fragment ACT017 (9012) which inhibits collagen-induced platelet aggregation in a dose-dependent manner. When tested in vivo in cynomolgus monkeys (Macaca fascicularis), the antibody derivative did not induce thrombocytopenia or deplete the receptor from platelets and it had no effect on normal hemostasis. ${ }^{83}$ Since phase I clinical trials in healthy volunteers proofed that ACT017 is a safe and well-tolerated compound showing favorable pharmacokinetic/pharmacodynamic properties, ${ }^{84}$ a phase II clinical trial in patients with AIS is currently in progress (NCT 03803007).

\section{Targeting CLEC-2 in Ischemic Stroke}

C-type lectin-like receptor 2 (CLEC-2) is a platelet receptor that contributes to platelet activation through a signaling pathway similar to GPVI. ${ }^{33,36}$ The only known ligands for CLEC-2 are the snake venom rhodocytin as well as the endogenous ligand podoplanin. ${ }^{39,85}$ On resting platelets CLEC-2 exists as a homo-dimerized receptor, which forms 
oligomers upon ligand binding. ${ }^{86}$ Contrary to GPVI-induced signaling, the initial phosphorylation of the central tyrosine in the hem-ITAM of CLEC-2 is mediated by the tyrosine kinase Syk and not Fyn or Lyn, which act more downstream in this signaling cascade, that otherwise uses similar effector and adapter molecules as GPVI, resulting in strong platelet activation. ${ }^{87}$ The interaction of podoplanin on lymphatic endothelial cells and neuroepithelial cells with CLEC-2 is essential to maintain the separation of lymphatic and blood vessels and prevent ICH during development, respectively. ${ }^{88,89}$ Therefore, global CLEC-2 deficiency in mice is lethal at the embryonic or neonatal stage. Those mice exhibit a disorganized vessel structure and impaired lymphatic clearance with blood-lymphatic mixing as well as impaired vascularization of the brain. In these processes, CLEC-2dependent signaling in platelets seems to be critical, since MK/platelet-specific deletion of CLEC-2, Syk, or SLP-76 resulted in the same blood-lymphatic mixing phenotype and hemorrhaging within the brain as the constitutive knockout of CLEC-2. ${ }^{90,91}$

It is likely that this receptor might also play an important role in hemostasis and thrombosis, because platelets are fully activated and form aggregates upon stimulation with the CLEC-2 agonist rhodocytin and form thrombi on podoplanincoated surfaces under flow. ${ }^{39,85,92}$ Indeed, platelet-specific loss of CLEC-2 upon treatment of mice with the antibody INU1 resulted in significant defects in platelet aggregate formation under flow in vitro, which translated into defective thrombus formation in a model of $\mathrm{FeCl}_{3}$-induced mesenteric artery injury and into prolonged bleeding times. ${ }^{93}$ Later on, the involvement of CLEC-2 in thrombus formation ex vivo and in vivo was corroborated in a study using fetal liver cell chimeric mice, with a genetic CLEC-2 deficiency, ${ }^{88}$ while another study found no significant role of CLEC-2 for platelet aggregate formation at arteriolar shear. ${ }^{94}$ More recently, a critical role for CLEC-2 was also observed in a mouse model of deep vein thrombosis, where the platelet specific knockout of the receptor prevented full vessel occlusion. ${ }^{95}$ Of note, bleeding times in those mice were not significantly altered compared with the corresponding controls.

Overall, these studies have provided compelling evidence that CLEC-2 contributes to platelet function in hemostasis and thrombosis, making it a potential antithrombotic target. Interestingly, this function of CLEC-2 appears to be independent of its hemITAM, ${ }^{96}$ indicating that it acts more as an adhesion receptor rather than a receptor for platelet activation in this setting.

Although pharmacological blockade of CLEC-2 appears to be a promising antithrombotic strategy, caution is warranted since the combined loss of GPVI and CLEC-2 leads to dramatically prolonged bleeding times as well as to abrogated thrombus formation, demonstrating functional redundancy of the two (hem)ITAM receptors. ${ }^{97}$ Furthermore, recent studies have suggested a complex role of CLEC-2 beyond classical hemostasis (reviewed in Rayes et al. ${ }^{33}$ ), e.g., in maintaining vascular integrity under inflammatory conditions, ${ }^{98,99}$ and in addition it was demonstrated that the podoplanin-CLEC-2 axis inhibits inflammation in mouse models of sepsis and lung injury. ${ }^{100,101}$ Therefore, the potential targeting of CLEC-2 in patients must be considered very carefully. Given that no patients with CLEC-2 deficiency are known to date, the relevance of this receptor in ischemic stroke in human might be questionable and needs further investigation, especially because there are no experimental studies addressing the role of CLEC- 2 in stroke. Nonetheless, plasma levels of soluble CLEC-2 (sCLEC-2) could recently be positively correlated with stroke progression, recurrence of vascular events, and risk of death in patients with AIS, making sCLEC-2 a promising prognostic marker for AIS $\left(-\right.$ Table 1). ${ }^{102,103}$

\section{FXII-Bradykinin in Ischemic Stroke}

Another hallmark of platelet activation besides the conformational change of GPIIb/IIIa to its high affinity state is the release of granules which store a plethora of mediators that further augment thrombotic and inflammatory processes. Among those, negatively charged inorganic PolyPs have a key role in the initiation of both coagulation and inflammation ${ }^{40}$ ( - Fig. 1). Upon contact with PolyP, coagulation factor FXII is turned into FXIIa representing the starting point of the intrinsic coagulation pathway. In addition, FXIIa cleaves plasma kallikrein (PK) which by itself activates FXII. ${ }^{40}$ Furthermore, the activated KKS leads to the liberation of the pro-inflammatory mediator bradykinin, which contributes to vessel dilatation, neutrophil recruitment, and increased vascular permeability upon binding to $\mathrm{B} 1$ and $\mathrm{B} 2$ receptors (B1R and B2R) on endothelial cells and neurons. ${ }^{40,104}$ Thus, the contact pathway provides a link between platelet-mediated activation of the intrinsic coagulation pathway via FXII and pro-inflammatory processes involving the KKS ( - Fig. 1). It was long believed that FXII is dispensable for blood clot formation since patients with hereditary deficiency of FXII display no bleeding phenotype, neither do mice with genetic knockout of FXII. However, FXII-deficient mice were protected from vessel occlusion in different in vivo models of arterial thrombosis as well as from collagen/epinephrineinduced pulmonary thromboembolism, showing that FXII is critical for pathological thrombus formation but not for hemostasis. ${ }^{105}$ Importantly, FXII-deficient mice or wild-type mice treated with the specific FXIla-inhibitor recombinant human albumin-Infestin-4 (rHA-Infestin-4) showed marked protection from ischemic brain injury in the TMCAO model without signs of ICH. ${ }^{106,107}$ These results suggest that FXII could be a promising therapeutic target for the treatment of AIS patients. Of note, a small cohort study of 22 patients suffering from ischemic stroke showed a significant activation of the tissue KKS, ${ }^{108}$ implicating that FXII might also be involved in stroke development in humans. The link of FXII to the pro-inflammatory KKS is further highlighted by studies investigating B1R, FXIla, and the PK blocker C1-inhibitor in the context of AIS. Mice deficient for B1R displayed smaller infarct sizes, less edema formation, better neurological outcome, and increased long-term survival compared with wild-type mice when subjected to the tMCAO model. Furthermore, ameliorated post-ischemic 
inflammation, reflected by decreased infiltration of neutrophilic granulocytes, macrophages, and $\mathrm{T}$ cells into the ischemic brain was seen in B1R-deficient mice. Similar results were obtained by pharmacological blockade of B1R with R715 in a dose-dependent manner. ${ }^{109}$ The $\mathrm{C} 1$-inhibitor is a serine protease classically known as a physiological inhibitor of the complement system. Moreover, it can block FXIIa and $\mathrm{PK}$, thereby preventing leukocyte recruitment during inflammation. ${ }^{110}$ In the tMCAO model, mice and rats treated with recombinant human C1-inhibitor (rhC1-inhibitor) 1 hour after induction of stroke showed significantly reduced infarct volumes, better neurological outcome, and improved survival ( - Table 1 ). This protective effect was mostly attributable to the protection of blood-brain barrier function reflected by less edema formation, attenuated inflammation, and prevention of tissue damage-triggered, secondary microvascular thrombosis. ${ }^{111}$ Furthermore, directly interfering with the KKS system by constitutively knocking out $\mathrm{PK}$ in mice $\left(\mathrm{PK}^{--}\right)$had favorable effects in the tMCAO model. Twenty-four hours after removal of the filament and reperfusion, $\mathrm{PK}^{-1-}$ mice were protected from brain infarction and showed improved neurological outcome, which was reverted when the animals had been reconstituted with human PK. The same was true for pharmacological interference with PK by administration of a neutralizing PK-specific antibody. Like C1-inhibitor-treated mice, PK-deficient mice showed similar anti-thrombotic and anti-inflammatory effects as well as no significant intracranial bleedings. ${ }^{112}$ These results support the concept that both thrombotic and inflammatory pathways operate in concert and are mechanistically linked to drive neuronal damage in the setting of AIS.

The other principal substrate of FXIIa is FXI, which in turn activates the intrinsic pathway of coagulation. Pretreatment of mice with the antibody 14E11, which inhibits activation of FXI by FXIIa, resulted in improved reperfusion, reduced infarct sizes, and less fibrin accumulation compared with untreated mice following tMCAO, without inducing $\mathrm{ICH} .{ }^{113}$ This further underlines the relevance of FXII in AIS, making it a druggable and potentially a safe target to combat AIS. However, clinical data on the relevance of FXI and FXII in patients in the context of ischemic stroke are not yet conclusive. Some studies showed higher FXI levels in patients with acute stroke and a correlation with poorer prognosis. ${ }^{114}$ Others could not find any differential regulation of FXI or FXII levels between different subtypes of acute cerebrovascular events, ${ }^{115}$ making FXI or FXII poor biomarkers to identify patients at risk of ischemic stroke. Nonetheless, experimental data suggest that the FXII-kallikrein-kinin pathway might be a safe molecular target to reduce brain damage in the setting of AIS.

Trials to elucidate the potential of interfering with the FXII-kallikrein-kinin pathway to treat AIS patients are currently ongoing $(-$ Table 1$)$. In addition, a phase II clinical trial deals with direct inhibition of FXIa by oral administration of BMS-986177 in combination with aspirin and clopidogrel to prevent recurrence of thrombotic events leading to secondary stroke in ischemic stroke patients (NCT03766581).

\section{Conclusions}

While the central function of platelets in hemostasis and thrombosis has been firmly established for decades, there is now an increasing awareness that these cells orchestrate a large variety of other physiological and pathological processes, most notably inflammation and innate immunity. Thereby, mechanisms and receptor functions that are critical in thrombosis and hemostasis are not necessarily suitable targets in thrombo-inflammatory diseases ( - Fig. 1 and - Table 1 ), highlighting the need to carefully assess efficacy of anti-platelet drugs in the respective experimental and clinical settings.

GPIb as well as GPVI have emerged as promising and potentially safe therapeutic targets in the treatment of thrombosis as well as AIS (-Table 1). Pharmacologic interference with these receptors improved neurological outcome and reduced the inflammatory response in experimental stroke models without increasing the bleeding risk.

One central conclusion of several preclinical stroke studies may be that the thrombotic occlusion of the microvasculature in the reperfused brain territory is likely the consequence rather than the cause of ischemic tissue damage. This is strongly supported by the observation that inhibition of platelet aggregation is not protective in these models, despite abrogating microvascular thrombosis. Based on this hypothesis, one might predict that more or less all approaches aiming at the inhibition of classical platelet-dependent thrombus formation may prevent or reduce the initial occurrence of stroke but not the resultant I/RI. Interfering with the thrombo-inflammatory activity of platelets might on the other hand provide protection from infarct progression without the major side effect of existing anti-thrombotic agents, namely the increased bleeding risk. $^{50-53}$ Of note, the exact contribution of platelet receptors and signaling pathways to inflammation and maintenance of vascular integrity seems to be determined by the affected tissue and vasculature as well as the pathogenic stimulus. ${ }^{99}$ This means that approaches leading to an improved outcome in AIS patients will not necessarily show beneficial effects in other thrombo-inflammatory disease settings affecting other organs.

Clearly, further studies are needed to better understand the mechanistic implications as well as the tissue- and stimulus-specific functions of platelet receptors, signaling pathways, and effector function in thrombo-inflammatory disease conditions. This will be of pivotal importance for finding safe and effective treatment strategies for patients with thrombotic and/or thrombo-inflammatory disorders.

Conflict of Interest

The authors declare that they have no conflict of interest.

\section{Acknowledgment}

The work was supported by the Deutsche Forschungsgemeinschaft (project number 374031971-CRC/TR 240).

\section{References}

1 Cameron SJ, Mix DS, Ture SK, et al. Hypoxia and ischemia promote a maladaptive platelet phenotype. Arterioscler Thromb Vasc Biol 2018;38(07):1594-1606 
2 Nieswandt B, Pleines I, Bender M. Platelet adhesion and activation mechanisms in arterial thrombosis and ischaemic stroke. J Thromb Haemost 2011;9(Suppl 1):92-104

3 Chung T, Connor D, Joseph J, et al. Platelet activation in acute pulmonary embolism. J Thromb Haemost 2007;5(05):918-924

4 Jackson SP. Arterial thrombosis-insidious, unpredictable and deadly. Nat Med 2011;17(11):1423-1436

5 Franks ZG, Campbell RA, Weyrich AS, Rondina MT. Plateletleukocyte interactions link inflammatory and thromboembolic events in ischemic stroke. Ann N Y Acad Sci 2010;1207:11-17

6 Grommes J, Alard JE, Drechsler M, et al. Disruption of plateletderived chemokine heteromers prevents neutrophil extravasation in acute lung injury. Am J Respir Crit Care Med 2012;185 (06):628-636

7 Nieswandt B, Kleinschnitz C, Stoll G. Ischaemic stroke: a thrombo-inflammatory disease? J Physiol 2011;589(17): 4115-4123

8 Feigin VL, Abajobir AA, Abate KH, et al; GBD 2015 Neurological Disorders Collaborator Group. Global, regional, and national burden of neurological disorders during 1990-2015: a systematic analysis for the Global Burden of Disease Study 2015. Lancet Neurol 2017;16(11):877-897

9 Feigin VL, Lawes CM, Bennett DA, Anderson CS. Stroke epidemiology: a review of population-based studies of incidence, prevalence, and case-fatality in the late 20th century. Lancet Neurol 2003;2(01):43-53

10 Saver JL, Gornbein J, Grotta J, et al. Number needed to treat to benefit and to harm for intravenous tissue plasminogen activator therapy in the 3- to 4.5-hour window: joint outcome table analysis of the ECASS 3 trial. Stroke 2009;40(07):2433-2437

11 de Los Ríos la Rosa F, Khoury J, Kissela BM, et al. Eligibility for intravenous recombinant tissue-type plasminogen activator within a population: the effect of the European Cooperative Acute Stroke Study (ECASS) III Trial. Stroke 2012;43(06):1591-1595

12 Leischner H, Flottmann F, Hanning U, et al. Reasons for failed endovascular recanalization attempts in stroke patients. J Neurointerv Surg 2019;11(05):439-442

13 Tsivgoulis G, Goyal N, Mikulik R, et al. Eligibility for mechanical thrombectomy in acute ischemic stroke: a phase IV multi-center screening log registry. J Neurol Sci 2016;371:96-99

14 Nour M, Scalzo F, Liebeskind DS. Ischemia-reperfusion injury in stroke. Intervent Neurol 2013;1(3-4):185-199

15 Mizuma A, You JS, Yenari MA. Targeting reperfusion injury in the age of mechanical thrombectomy. Stroke 2018;49(07): 1796-1802

16 Eltzschig HK, Eckle T. Ischemia and reperfusion-from mechanism to translation. Nat Med 2011;17(11):1391-1401

17 Braeuninger S, Kleinschnitz C, Nieswandt B, Stoll G. Focal cerebral ischemia. Methods Mol Biol 2012;788:29-42

18 Kleinschnitz C, Schwab N, Kraft P, et al. Early detrimental T-cell effects in experimental cerebral ischemia are neither related to adaptive immunity nor thrombus formation. Blood 2010;115 (18):3835-3842

19 Stoll G, Nieswandt B. Thrombo-inflammation in acute ischaemic stroke - implications for treatment. Nat Rev Neurol 2019;15(08): 473-481

20 Zrzavy T, Machado-Santos J, Christine S, et al. Dominant role of microglial and macrophage innate immune responses in human ischemic infarcts. Brain Pathol 2018;28(06):791-805

21 Yilmaz G, Arumugam TV, Stokes KY, Granger DN. Role of T lymphocytes and interferon-gamma in ischemic stroke. Circulation 2006;113(17):2105-2112

22 Caudrillier A, Kessenbrock K, Gilliss BM, et al. Platelets induce neutrophil extracellular traps in transfusion-related acute lung injury. J Clin Invest 2012;122(07):2661-2671

23 McDonald B, Urrutia R, Yipp BG, Jenne CN, Kubes P. Intravascular neutrophil extracellular traps capture bacteria from the bloodstream during sepsis. Cell Host Microbe 2012;12(03):324-333
24 Strecker J-K, Schmidt A, Schäbitz W-R, Minnerup J. Neutrophil granulocytes in cerebral ischemia - evolution from killers to key players. Neurochem Int 2017;107:117-126

25 Obrenovitch TP, Hallenbeck JM. Platelet accumulation in regions of low blood flow during the postischemic period. Stroke 1985; 16(02):224-234

26 Del Zoppo GJ, Copeland BR, Harker LA, et al. Experimental acute thrombotic stroke in baboons. Stroke 1986;17(06):1254-1265

27 Sandercock PA, Counsell C, Tseng MC, Cecconi E. Oral antiplatelet therapy for acute ischaemic stroke. Cochrane Database Syst Rev 2014;(03):CD000029

28 Zinkstok SM, Beenen LF, Majoie CB, Marquering HA, de Haan RJ, Roos YB. Early deterioration after thrombolysis plus aspirin in acute stroke: a post hoc analysis of the Antiplatelet Therapy in Combination with Recombinant t-PA Thrombolysis in Ischemic Stroke trial. Stroke 2014;45(10):3080-3082

29 Savage B, Saldívar E, Ruggeri ZM. Initiation of platelet adhesion by arrest onto fibrinogen or translocation on von Willebrand factor. Cell 1996;84(02):289-297

30 Kanaji S, Fahs SA, Shi Q Haberichter SL, Montgomery RR. Contribution of platelet vs. endothelial VWF to platelet adhesion and hemostasis. J Thromb Haemost 2012;10(08):1646-1652

31 Moroi M, Jung SM, Nomura S, Sekiguchi S, Ordinas A, Diaz-Ricart M. Analysis of the involvement of the von Willebrand factorglycoprotein Ib interaction in platelet adhesion to a collagencoated surface under flow conditions. Blood 1997;90(11): 4413-4424

32 Mammadova-Bach E, Ollivier V, Loyau S, et al. Platelet glycoprotein VI binds to polymerized fibrin and promotes thrombin generation. Blood 2015;126(05):683-691

33 Rayes J, Watson SP, Nieswandt B. Functional significance of the platelet immune receptors GPVI and CLEC-2. J Clin Invest 2019; 129(01):12-23

34 Alshehri OM, Hughes CE, Montague S, et al. Fibrin activates GPVI in human and mouse platelets. Blood 2015;126(13):1601-1608

35 Braun A, Vogtle T, Varga-Szabo D, Nieswandt B. STIM and Orai in hemostasis and thrombosis. Front Biosci 2011;16:2144-2160

36 Bergmeier W, Stefanini L. Platelet ITAM signaling. Curr Opin Hematol 2013;20(05):445-450

37 Heemskerk JW, Mattheij NJ, Cosemans JM. Platelet-based coagulation: different populations, different functions. J Thromb Haemost 2013;11(01):2-16

38 Stegner D, Nieswandt B. Platelet receptor signaling in thrombus formation. J Mol Med (Berl) 2011;89(02):109-121

39 Suzuki-Inoue K, Kato Y, Inoue O, et al. Involvement of the snake toxin receptor CLEC-2, in podoplanin-mediated platelet activation, by cancer cells. J Biol Chem 2007;282(36):25993-26001

40 Müller F, Mutch NJ, Schenk WA, et al. Platelet polyphosphates are proinflammatory and procoagulant mediators in vivo. Cell 2009; 139(06):1143-1156

41 Coller BS. Anti-GPIIb/IIIa drugs: current strategies and future directions. Thromb Haemost 2001;86(01):427-443

42 Neri M, Riezzo I, Pascale N, Pomara C, Turillazzi E. Ischemia/reperfusion injury following acute myocardial infarction: a critical issue for clinicians and forensic pathologists. Mediators Inflamm 2017;2017:7018393

43 Safley DM, Venkitachalam L, Kennedy KF, Cohen DJ. Impact of glycoprotein IIb/IIIa inhibition in contemporary percutaneous coronary intervention for acute coronary syndromes: insights from the National Cardiovascular Data Registry. JACC Cardiovasc Interv 2015;8(12):1574-1582

44 Barrabés JA, Inserte J, Mirabet M, et al. Antagonism of P2Y12 or GPIIb/IIla receptors reduces platelet-mediated myocardial injury after ischaemia and reperfusion in isolated rat hearts. Thromb Haemost 2010;104(01):128-135

45 Cannon CP. Combination therapy for acute myocardial infarction: glycoprotein IIb/IIIa inhibitors plus thrombolysis. Clin Cardiol 1999;22(8, Suppl):IV37-IV43 
46 Montalescot G, Barragan P, Wittenberg O, et al; ADMIRAL Investigators. Abciximab before Direct Angioplasty and Stenting in Myocardial Infarction Regarding Acute and Long-Term Followup. Platelet glycoprotein IIb/IIIa inhibition with coronary stenting for acute myocardial infarction. N Engl J Med 2001;344(25): 1895-1903

47 Choudhri TF, Hoh BL, Zerwes HG, et al. Reduced microvascular thrombosis and improved outcome in acute murine stroke by inhibiting GP IIb/IIIa receptor-mediated platelet aggregation. J Clin Invest 1998;102(07):1301-1310

48 Abumiya T, Fitridge R, Mazur C, et al. Integrin alpha(IIb)beta(3) inhibitor preserves microvascular patency in experimental acute focal cerebral ischemia. Stroke 2000;31(06):1402-1409, discussion $1409-1410$

49 Lapchak PA, Araujo DM, Song D, Zivin JA. The nonpeptide glycoprotein IIb/IIIa platelet receptor antagonist SM-20302 reduces tissue plasminogen activator-induced intracerebral hemorrhage after thromboembolic stroke. Stroke 2002;33(01): $147-152$

50 Kleinschnitz C, Pozgajova M, Pham M, Bendszus M, Nieswandt B, Stoll G. Targeting platelets in acute experimental stroke: impact of glycoprotein Ib, VI, and IIb/IIla blockade on infarct size, functional outcome, and intracranial bleeding. Circulation 2007;115(17):2323-2330

51 Kraft P, Schuhmann MK, Fluri F, et al. Efficacy and safety of platelet glycoprotein receptor blockade in aged and comorbid mice with acute experimental stroke. Stroke 2015;46(12): 3502-3506

52 Adams HP Jr, Effron MB, Torner J, et al; AbESTT-II Investigators. Emergency administration of abciximab for treatment of patients with acute ischemic stroke: results of an international phase III trial: Abciximab in Emergency Treatment of Stroke Trial (AbESTT-II). Stroke 2008;39(01):87-99

53 Kellert L, Hametner C, Rohde S, et al. Endovascular stroke therapy: tirofiban is associated with risk of fatal intracerebral hemorrhage and poor outcome. Stroke 2013;44(05):1453-1455

54 Zhu YQ Zhang YJ, Ruan HL, Liu Q, Zhan Q Li Q. Safety of tirofiban for patients with acute ischemic stroke in routine clinical practice. Exp Ther Med 2015;10(01):169-174

55 Chang Y, Kim BM, Bang OY, et al. Rescue stenting for failed mechanical thrombectomy in acute ischemic stroke: a multicenter experience. Stroke 2018;49(04):958-964

56 Pham M, Helluy X, Kleinschnitz C, et al. Sustained reperfusion after blockade of glycoprotein-receptor-Ib in focal cerebral ischemia: an MRI study at 17.6 Tesla. PLoS One 2011;6(04):e18386

57 Schuhmann MK, Guthmann J, Stoll G, Nieswandt B, Kraft P, Kleinschnitz C. Blocking of platelet glycoprotein receptor Ib reduces "thrombo-inflammation" in mice with acute ischemic stroke. J Neuroinflammation 2017;14(01):18

58 Kanaji T, Russell S, Ware J. Amelioration of the macrothrombocytopenia associated with the murine Bernard-Soulier syndrome. Blood 2002;100(06):2102-2107

59 De Meyer SF, Schwarz T, Schatzberg D, Wagner DD. Platelet glycoprotein Ib $\alpha$ is an important mediator of ischemic stroke in mice. Exp Transl Stroke Med 2011;3(01):9

60 Kleinschnitz C, De Meyer SF, Schwarz T, et al. Deficiency of von Willebrand factor protects mice from ischemic stroke. Blood 2009;113(15):3600-3603

61 Verhenne S, Denorme F, Libbrecht S, et al. Platelet-derived VWF is not essential for normal thrombosis and hemostasis but fosters ischemic stroke injury in mice. Blood 2015;126(14):1715-1722

62 Zhao BQ Chauhan AK, Canault M, et al. von Willebrand factorcleaving protease ADAMTS13 reduces ischemic brain injury in experimental stroke. Blood 2009;114(15):3329-3334

63 Fujioka M, Hayakawa K, Mishima K, et al. ADAMTS13 gene deletion aggravates ischemic brain damage: a possible neuroprotective role of ADAMTS13 by ameliorating postischemic hypoperfusion. Blood 2010;115(08):1650-1653
64 Canobbio I, Balduini C, Torti M. Signalling through the platelet glycoprotein Ib-V-IX complex. Cell Signal 2004;16(12):1329-1344

65 Elvers M, Stegner D, Hagedorn I, et al. Impaired alpha(IIb)beta(3) integrin activation and shear-dependent thrombus formation in mice lacking phospholipase D1. Sci Signal 2010;3(103):ra1

66 Klier M, Gowert NS, Jäckel S, Reinhardt C, Elvers M. Phospholipase D1 is a regulator of platelet-mediated inflammation. Cell Signal 2017;38:171-181

67 Thielmann I, Stegner D, Kraft P, et al. Redundant functions of phospholipases D1 and D2 in platelet $\alpha$-granule release. J Thromb Haemost 2012;10(11):2361-2372

68 Stegner D, Thielmann I, Kraft P, Frohman MA, Stoll G, Nieswandt B. Pharmacological inhibition of phospholipase D protects mice from occlusive thrombus formation and ischemic stroke-brief report. Arterioscler Thromb Vasc Biol 2013;33(09):2212-2217

69 Maguire JM, Thakkinstian A, Sturm J, et al. Polymorphisms in platelet glycoprotein 1 balpha and factor VII and risk of ischemic stroke: a meta-analysis. Stroke 2008;39(06):1710-1716

70 Nieswandt B, Watson SP. Platelet-collagen interaction: is GPVI the central receptor? Blood 2003;102(02):449-461

71 Nieswandt B, Schulte V, Bergmeier W, et al. Long-term antithrombotic protection by in vivo depletion of platelet glycoprotein VI in mice. J Exp Med 2001;193(04):459-469

72 Massberg S, Gawaz M, Grüner S, et al. A crucial role of glycoprotein VI for platelet recruitment to the injured arterial wall in vivo. J Exp Med 2003;197(01):41-49

73 Lockyer S, Okuyama K, Begum S, et al. GPVI-deficient mice lack collagen responses and are protected against experimentally induced pulmonary thromboembolism. Thromb Res 2006;118 (03):371-380

74 Schuhmann MK, Kraft P, Bieber M, et al. Targeting platelet GPVI plus rt-PA administration but not $\alpha 2 \beta 1$-mediated collagen binding protects against ischemic brain damage in mice. Int J Mol Sci 2019;20(08):2019

75 van Eeuwijk JM, Stegner D, Lamb DJ, et al. The novel oral Syk inhibitor, Bl1002494, protects mice from arterial thrombosis and thromboinflammatory brain infarction. Arterioscler Thromb Vasc Biol 2016;36(06):1247-1253

76 Cherpokova D, Bender M, Morowski M, et al. SLAP/SLAP2 prevent excessive platelet (hem)ITAM signaling in thrombosis and ischemic stroke in mice. Blood 2015;125(01):185-194

77 Bigalke B, Stellos K, Geisler T, et al. Expression of platelet glycoprotein VI is associated with transient ischemic attack and stroke. Eur J Neurol 2010;17(01):111-117

78 Bender M, Hofmann S, Stegner D, et al. Differentially regulated GPVI ectodomain shedding by multiple platelet-expressed proteinases. Blood 2010;116(17):3347-3355

79 Montague SJ, Andrews RK, Gardiner EE. Mechanisms of receptor shedding in platelets. Blood 2018;132(24):2535-2545

80 Al-Tamimi M, Gardiner EE, Thom JY, et al. Soluble glycoprotein VI is raised in the plasma of patients with acute ischemic stroke. Stroke 2011;42(02):498-500

81 Ungerer M, Rosport K, Bültmann A, et al. Novel antiplatelet drug revacept (dimeric glycoprotein VI-Fc) specifically and efficiently inhibited collagen-induced platelet aggregation without affecting general hemostasis in humans. Circulation 2011;123(17): 1891-1899

82 Goebel S, Li Z, Vogelmann J, et al. The GPVI-Fc fusion protein Revacept improves cerebral infarct volume and functional outcome in stroke. PLoS One 2013;8(07):e66960

83 Lebozec K, Jandrot-Perrus M, Avenard G, Favre-Bulle O, Billiald P. Design, development and characterization of ACT017, a humanized Fab that blocks platelet's glycoprotein VI function without causing bleeding risks. MAbs 2017;9(06):945-958

84 Voors-Pette C, Lebozec K, Dogterom P, et al. Safety and tolerability, pharmacokinetics, and pharmacodynamics of ACT017, an antiplatelet GPVI (glycoprotein VI) Fab. Arterioscler Thromb Vasc Biol 2019;39(05):956-964 
85 Suzuki-Inoue K, Fuller GL, García A, et al. A novel Syk-dependent mechanism of platelet activation by the C-type lectin receptor CLEC-2. Blood 2006;107(02):542-549

86 Hughes CE, Pollitt AY, Mori J, et al. CLEC-2 activates Syk through dimerization. Blood 2010;115(14):2947-2955

87 Séverin S, Pollitt AY, Navarro-Nuñez L, et al. Syk-dependent phosphorylation of CLEC-2: a novel mechanism of hem-immunoreceptor tyrosine-based activation motif signaling. J Biol Chem 2011;286(06):4107-4116

88 Suzuki-Inoue K, Inoue O, Ding G, et al. Essential in vivo roles of the C-type lectin receptor CLEC-2: embryonic/neonatal lethality of CLEC-2-deficient mice by blood/lymphatic misconnections and impaired thrombus formation of CLEC-2-deficient platelets. J Biol Chem 2010;285(32):24494-24507

89 Lowe KL, Finney BA, Deppermann C, et al. Podoplanin and CLEC-2 drive cerebrovascular patterning and integrity during development. Blood 2015;125(24):3769-3777

90 Bertozzi CC, Schmaier AA, Mericko P, et al. Platelets regulate lymphatic vascular development through CLEC-2-SLP-76 signaling. Blood 2010;116(04):661-670

91 Finney BA, Schweighoffer E, Navarro-Núñez L, et al. CLEC-2 and Syk in the megakaryocytic/platelet lineage are essential for development. Blood 2012;119(07):1747-1756

92 Navarro-Núñez L, Pollitt AY, Lowe K, Latif A, Nash GB, Watson SP. Platelet adhesion to podoplanin under flow is mediated by the receptor CLEC-2 and stabilised by Src/Syk-dependent platelet signalling. Thromb Haemost 2015;113(05):1109-1120

93 May F, Hagedorn I, Pleines I, et al. CLEC-2 is an essential plateletactivating receptor in hemostasis and thrombosis. Blood 2009; 114(16):3464-3472

94 Hughes CE, Navarro-Núñez L, Finney BA, Mourão-Sá D, Pollitt AY, Watson SP. CLEC-2 is not required for platelet aggregation at arteriolar shear. J Thromb Haemost 2010;8(10):2328-2332

95 Payne H, Ponomaryov T, Watson SP, Brill A. Mice with a deficiency in CLEC-2 are protected against deep vein thrombosis. Blood 2017;129(14):2013-2020

96 Haining EJ, Cherpokova D, Wolf K, et al. CLEC-2 contributes to hemostasis independently of classical hemITAM signaling in mice. Blood 2017;130(20):2224-2228

97 Bender M, May F, Lorenz V, et al. Combined in vivo depletion of glycoprotein VI and C-type lectin-like receptor 2 severely compromises hemostasis and abrogates arterial thrombosis in mice. Arterioscler Thromb Vasc Biol 2013;33(05):926-934

98 Boulaftali Y, Hess PR, Getz TM, et al. Platelet ITAM signaling is critical for vascular integrity in inflammation. J Clin Invest 2013; 123(02):908-916

99 Rayes J, Jadoui S, Lax S, et al. The contribution of platelet glycoprotein receptors to inflammatory bleeding prevention is stimulus and organ dependent. Haematologica 2018;103(06): e256-e258

100 Rayes J, Lax S, Wichaiyo S, et al. The podoplanin-CLEC-2 axis inhibits inflammation in sepsis. Nat Commun 2017;8(01):2239
101 Lax S, Rayes J, Wichaiyo S, et al. Platelet CLEC-2 protects against lung injury via effects of its ligand podoplanin on inflammatory alveolar macrophages in the mouse. Am J Physiol Lung Cell Mol Physiol 2017;313(06):L1016-L1029

102 Zhang X, Zhang W, Wu X, et al. Prognostic significance of plasma CLEC-2 (C-type lectin-like receptor 2) in patients with acute ischemic stroke. Stroke 2018;50(01):A118022563

103 Wu X, Zhang W, Li H, et al. Plasma C-type lectin-like receptor 2 as a predictor of death and vascular events in patients with acute ischemic stroke. Eur J Neurol 2019;26(10):1334-1340

104 Müller F, Renné T. Novel roles for factor XII-driven plasma contact activation system. Curr Opin Hematol 2008;15(05): 516-521

105 Renné T, Pozgajová M, Grüner S, et al. Defective thrombus formation in mice lacking coagulation factor XII. J Exp Med 2005;202(02):271-281

106 Kleinschnitz C, Stoll G, Bendszus M, et al. Targeting coagulation factor XII provides protection from pathological thrombosis in cerebral ischemia without interfering with hemostasis. J Exp Med 2006;203(03):513-518

107 Hagedorn I, Schmidbauer S, Pleines I, et al. Factor XIIa inhibitor recombinant human albumin Infestin-4 abolishes occlusive arterial thrombus formation without affecting bleeding. Circulation 2010;121(13):1510-1517

108 Wagner S, Kalb P, Lukosava M, Hilgenfeldt U, Schwaninger M. Activation of the tissue kallikrein-kinin system in stroke. J Neurol Sci 2002;202(1-2):75-76

109 Austinat M, Braeuninger S, Pesquero JB, et al. Blockade of bradykinin receptor B1 but not bradykinin receptor B2 provides protection from cerebral infarction and brain edema. Stroke 2009;40(01):285-293

110 Davis AE III, Mejia P, Lu F. Biological activities of C1 inhibitor. Mol Immunol 2008;45(16):4057-4063

111 Heydenreich N, Nolte MW, Göb E, et al. C1-inhibitor protects from brain ischemia-reperfusion injury by combined antiinflammatory and antithrombotic mechanisms. Stroke 2012;43 (09):2457-2467

112 Göb E, Reymann S, Langhauser F, et al. Blocking of plasma kallikrein ameliorates stroke by reducing thromboinflammation. Ann Neurol 2015;77(05):784-803

113 Leung PY, Hurst S, Berny-Lang MA, et al. Inhibition of Factor XIImediated activation of factor XI provides protection against experimental acute ischemic stroke in mice. Transl Stroke Res 2012;3(03):381-389

114 Undas A, Slowik A, Gissel M, Mann KG, Butenas S. Circulating activated factor $\mathrm{XI}$ and active tissue factor as predictors of worse prognosis in patients following ischemic cerebrovascular events. Thromb Res 2011;128(05):e62-e66

115 Kraft P, Drechsler C, Gunreben I, Heuschmann PU, Kleinschnitz C. Regulation of blood coagulation factors XI and XII in patients with acute and chronic cerebrovascular disease: a case-control study. Cerebrovasc Dis 2014;38(05):337-343 